\title{
In Praise of Small Data Nancy Reid
}

\section{Introduction}

This paper is based on the Gibbs Lecture presented at the 2020 Joint Mathematical Meetings in Denver, Colorado. I began the lecture by acknowledging the honour of the invitation and thanking the American Mathematical Society for their confidence in me. I then gave a brief description of the use of the Gibbs distribution as a basis for a range of computational techniques that have been very influential in statistical science. I omit the description here, as it is well explained in, for example, Casella \& George (1992) and book-length treatments such as Brooks et al. (2011). The name "Gibbs sampler" was first used in Geman \& Geman (1984).

Statistical science has its roots in mathematics, but is quite a different field, and I am often asked by my mathematical colleagues "What's new in statistics?" For most of my career, the answer would likely have been some combination of a description of a particular theoretical problem I was working on at the time, and a vague statement about the vast increase in computer power having a big influence on the application of statistics in various fields. More recently, I would say the biggest news in statistics is that interest in the topic is exploding. Figure 1 shows the enrolment growth in students registered in statistics undergraduate programs at the University of Toronto over the past 25 years, showing a 10-fold growth in enrolments in the past 10 years. ${ }^{1}$ The University of Toronto is not special in this respect, just bigger; the news magazine of the American Statistical Association carried the headline in the December 2019 issue "(Bio)Statistics Bachelor's Degrees Nearly

Nancy Reid is university professor of statistical sciences at the University of Toronto. Her email address is reid@utstat. utoronto.ca.

Communicated by Notices Associate Editor Richard Levine.

For permission to reprint this article, please contact:

reprint-permission@ams.org.

DOI: https://doi.org/10.1090/noti2207

${ }^{1}$ A similar plot from 1977, the year of the formation of our department, to 1994 would be essentially flat at or below the level in 1994.

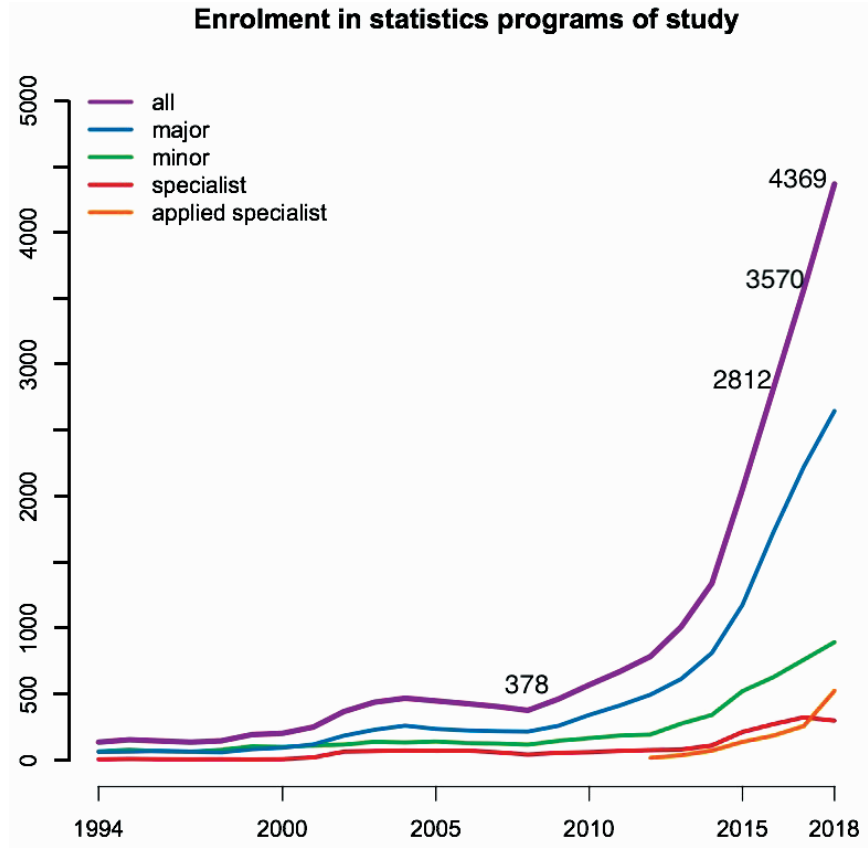

Figure 1. Enrolment in undergraduate programs in statistics at the University of Toronto, 1994-2018.

Quintuple This Decade," accompanied by a similar exponential graph. ${ }^{2}$

As both a consequence and perhaps a cause of this increased interest from students, the field is more than ever looking outward. Statistical science, both theory and applied, has always been motivated by practical problems, but the departmental structure at most universities often led to an artificial distinction between theoretical statistics, studied in departments of mathematics and statistics, and applied statistics, developed in cognate departments such as engineering, psychology, public health, and so on. The University of Toronto's Department of Statistical Sciences has been fortunate to be able to make a broad range of academic appointments in recent years, with joint appointments in sociology, philosophy, astronomy, psychology,

${ }^{2}$ Links to websites are live in the digital version of this article, and on the author's website. 
computer science, information science, and environmental science. As well, in common with most statistics departments, we have a long-standing tradition of joint appointments with biostatistics. I believe our experience reflects the North American trend of very large increases in undergraduate enrolments and corresponding pedagogical and curriculum innovations.

\section{Statistics and Data Science}

I think the beginning of this increased interest in statistical science can be traced to a front-page article in the New York Times in August 2009. The article quoted Hal Varian, then the chief economist at Google: "I keep saying that the sexy job in the next 10 years will be statisticians ... and I'm not joking." In June 2008 the editor-in-chief at Wired published, with perhaps less prescience, an article with the headline "The end of theory: the data deluge makes the scientific method obsolete." The media interest in so-called Big Data increased rapidly; the phrase reached a peak on the Gartner hype cycle in 2013, after which time it became something less novel and more routine, and has been replaced as a catch-all phrase by the broader but still vague term Data Science. In 2015, the Fields Institute for Research in the Mathematical Sciences, along with the Canadian Statistical Sciences Institute, the Centre de recherches mathématiques, and the Pacific Institute for the Mathematical Sciences, held a semester-long program called "Statistical inference, learning and models for big data"; the retrospective workshop in 2018 was "Statistical inference, learning and models for data science," reflecting the change of emphasis in the intervening three years. ${ }^{3}$ There are now dozens, if not hundreds, of data science institutes and training programs around the world, although I don't think it is quite clear yet if data science is a field of research, or a set of principles and strategies that inform other fields of research. The talk and subsequent paper by Donoho (2017) has been especially influential. Meng (2019) describes data science as an ecosystem.

Statisticians have spent a good deal of time considering the relationship between statistical science and data science. For a broad outline of the practice of statistical science one can hardly do better than to follow Cox \& Donnelly (2011, Ch. 2). One starts with a scientific question, ${ }^{4}$ assesses how data might shed light on this question, and plans data collection, considering carefully potential sources of variation and of bias, and designing the data collection to minimize their impact. These initial steps necessarily involve close collaboration with the scientists, although in point of fact are often carried out very skilfully

\footnotetext{
${ }^{3}$ Talks at both these events are available for viewing at FieldsLive.

${ }^{4}$ I am using "scientific" to mean any field of study, including the social sciences and, increasingly, the humanities.
}

by scientists without input from statisticians. However, statisticians are very well-trained in thinking about how things might go wrong, so close collaboration between subject-matter experts and statisticians may well be beneficial. Statistical science provides strategies for analysis of the data: modelling, computation, inferential methods, and so on. This is a large part of what is taught in graduate and undergraduate programs, often with less emphasis on the earlier planning stages. The more mathematical part of statistical science is concerned with the properties of statistical methods. Sometimes this involves evaluating methods that have been developed by ad hoc means for use in particular applications-one goal being to understand whether or not these methods can be relied upon. Statistical theory develops general principles to guide these assessments, and to study how they may be generalized for a broader range of applications. In the 1950s there was a concerted effort to develop optimal statistical methods from first principles, sometimes described as an attempt to axiomatize statistics, but this did not turn out to be very fruitful for the increasing number of application areas needing statistical methods for their scientific work. Traditional undergraduate and graduate training spends a good deal more time on the mathematical aspects of the discipline than the full set of applied statistics research components outlined by Cox \& Donnelly (2011). While it is recognized that communication of statistical results through plain-language summaries and high-quality visualizations is an important part of the discipline, it has not been much emphasized in many statistics programs.

The emergence of data science is at least partly a reaction to this narrowness of traditional statistical training as well as a reaction to the explosion in available data in nearly every scientific discipline. There are innumerable charts illustrating "data science workflow," some helpful and many hype-full, but the main point is to emphasize the entire process: from sourcing the data through to a data-driven policy decision. This includes at least the components in Figure 2, which lists what I think are the main elements of data science. Most diagrams of this type also include a series of (circular) arrows to suggest the "flow" aspect of the work.

The data acquisition element refers to finding (or creating) the data needed to answer a scientific question. Some aspects of this are core parts of traditional training in statistics, which has long emphasized the importance of reliable and efficient methods for data collection, and the importance of assessing the quality and provenance of data. Statisticians' training in skepticism about data sources is often useful with big data, and is typically absent from training in computer science. On the other hand, the management and storage of data, especially very large volumes 


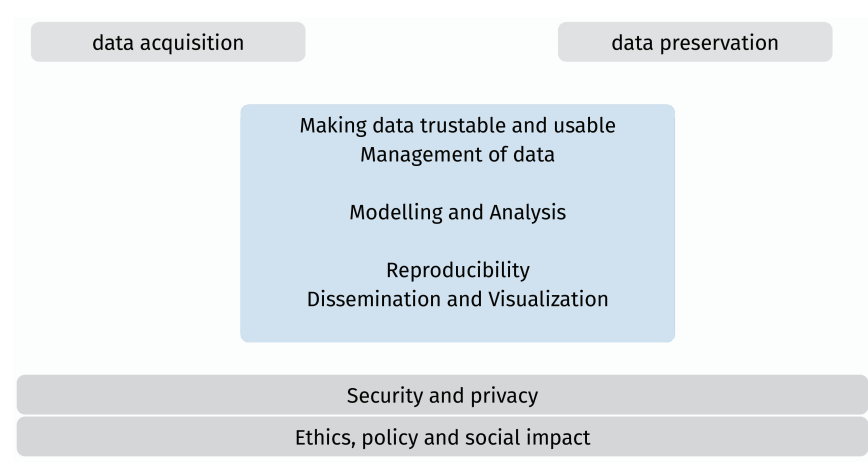

Figure 2. Example of a data science workflow.

of data, possibly being collected nearly continuously, requires expertise from computer science. Data preservation is commonly associated with information science or library science, but includes as well data repositories established in various fields of science, and in that case requires expertise from the subject-matter scientists as well.

A great deal of statistical training and research is concentrated on the modelling and analysis component. Statistical science tends to emphasize interpretable, often simple, models. In contrast, machine learning tends to be more concerned about good predictions, and as a result the models can be very complex. A reviewer quite correctly pointed out that exploration of the data, typically with plots and simple summaries, could well be included before the modelling and analysis component, and often provides the basis for dissemination and visualization later in the process. Reproducibility is a relatively new topic, prominent in data science courses and programs. Dissemination of analytic results or machine learning predictions, which usually includes some emphasis on visualization, can refer to anything from refereed journal articles to position papers to blog posts to media for public consumption. The best media outlets publish reasonably clear summaries of scientific results, often with very accomplished statistical graphics. Statistical graphics is somewhat distinct from the more general topic of information visualization; the latter is usually associated with computer science and in particular human-computer interaction. Visualization is an essential component of data science, whereas in traditional training it was perhaps construed as a one-hour add-on to a course in applied statistics. Reproducibility and visualization are discussed in more detail in $\$ 4$.

Recognition of security and privacy as critical components of data science is widespread, as people become aware of how much data about them is routinely collected from their digital life. Differential privacy, a technical subject developed in computer science, and comprising much statistical and mathematical sophistication, is one approach to enabling secure processing of data (Dwork et al., 2017; Reiter, 2019). The 2020 US Census has decided to use differential privacy for the first time in releasing its data products (Hawes, 2020). Government statistical agencies have for many years used various versions of disclosure limitation, along with restricted access to data, to ensure privacy of information collected in censuses and in surveys. Health records are typically secured using some version of deidentification or anonymization, although there is disagreement on the effectiveness of this, especially when several data bases can be linked through common elements. Computer science, statistics, and mathematics are all involved in various aspects of privacy and security.

The two bottom elements in Figure 2 require collaboration with the social sciences and humanities. Although sketched here as following the other components, this is inadequate, as they are important in all stages of the work. Questions about the fairness and transparency of complex algorithms has emerged as a pressing concern, particularly when these algorithms are used to decide high-impact outcomes for individuals, such as securing a loan, or being released on bail. A very readable popular account is $\mathrm{O}^{\prime} \mathrm{Neil}$ (2016). Rudin et al. (2020) make a compelling case for requiring increased transparency of black-box algorithms, with special reference to the COMPAS model used in the US for scoring risk of recidivism. The six commentaries to Rudin et al. (2020) add valuable counterpoints, extensions, and further references. Professional statistical organizations have codes of conduct for ethical use of statistical methods, but these were developed in the era of small data, and need adapting to the world of artificial intelligence and machine learning.

The elements of Figure 2 have been described as proceeding linearly in time, but in practice there needs to be interplay and feedback among the various elements. The elements in grey are meant to be cross-cutting components that inform the whole process.

This brief summary of my selection of components of data science involves much broader collaboration than was customary in the last century. Success in implementing them requires a combination of expertise in computer science, statistics, mathematics, the social sciences, and humanities. There is another set of essential collaboratorsthe domain scientists whose data is informing progress in so many areas. Statistics and data science as fields of study are meaningless without continual interaction with data that are used to advance knowledge. In this respect they are quite different from pure mathematics, which can be and has been very fruitfully developed as a closed system. This is not to deny the remarkable impact that fundamental mathematical discoveries have had in other sciencesnumber theory for example forming a foundation for 
cryptography, or topology for studying astronomical objects. But statistical and data science take their inspiration from, and register their impact on, scientific collaboration.

\section{Statistics in the News}

In order to illustrate this fruitful interplay between statistical science, computer science, mathematics, and domain science, I have selected, quite arbitrarily, examples of news articles from the past several months in which data collection and analysis played a key role.

3.1. Wildfire and climate change. On January 8, 2019, the Canadian newspaper The Globe and Mail featured an article with the headline "B.C. wildfires stoked by climate change, likely to become worse: study." The article referred to a study then recently published in the journal Earth's Future (Kirchmeier-Young et al., 2019) which used the theory of attribution analysis to study whether the severity of the 2017 fire season in British Columbia could plausibly be attributed to human-induced climate change. The statistical methods used in the paper drew on a 2016 consensus report from the National Academies, which described various methods for attribution of individual weather events to climate change. The description of the scope of the report notes

The relatively young science of extreme event attribution seeks to tease out the influence of humancaused climate change from other factors, such as natural sources of variability like El Niño, as contributors to individual extreme events.

While individual extreme events such as hurricanes, wildfires, and flooding are often attributed to climate change in the popular press, this is rarely a direct connection, and often difficult to establish rigorously. One of the outcome measures investigated by Kirchmeier-Young et al. (2019) was the area burned, and one of their conclusions was that "anthropogenic climate change increased the area burned by a factor of 7-11." The figure accompanying this conclusion is reproduced here in Figure 3. The plot on the left shows a time series of the predicted size of wildfires in their study area in southwestern British Columbia, from 1960 to 2020. There are 50 such time series, in grey, constructed from 50 simulations of a regional climate model. The bold black line shows the average of the 50 modelled time series. The area burned in 2017 was 1.2 million hectares, indicated by the dotted line across the top of the left figure. The decades 1960-1970 and 2010-2020 are highlighted in that plot with blue and red boxes. From these two 10-year windows a probability density for wildfire size was estimated using well-established nonparametric techniques for density estimation. The two estimated densities are plotted on the right, where the size of the fires is now recorded on

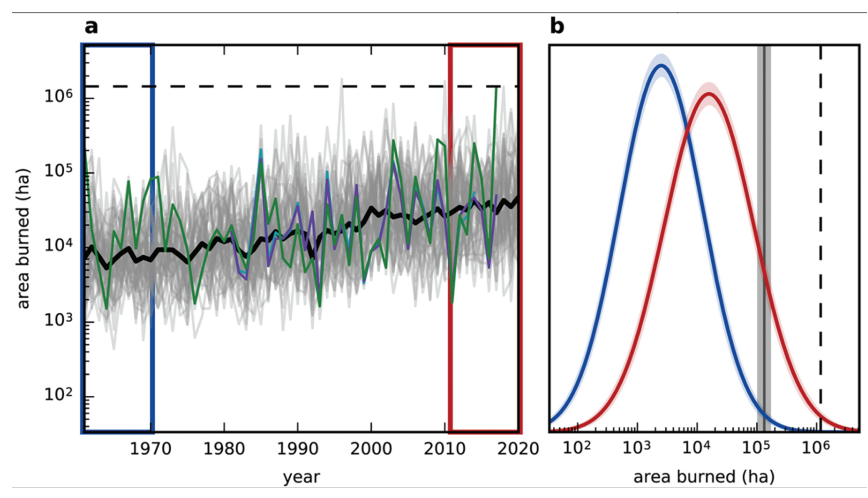

Figure 3. Part of Figure 5 of Kirchmeier-Young et al. (2019). The left graph shows 50 simulated climate scenarios (grey), the mean of these (black) and the observations (green). The $y$-axis shows the area burned, as predicted by a regression model, and the $x$-axis shows time. The size of the 2017 fire is indicated by the dashed line just above $10^{6}$. The right graph shows a probability density estimate based on these simulations: one for 1960-1970 and a second for 2010-2020. These decades are marked in blue and red, respectively, on the left graph. The vertical dashed line shows the fire in 2017, and the shaded grey line shows that percentile point in the 1960-1970 distribution, assumed to be free of anthropogenic influences. The 2017 fire was approximately 9 times larger on average than the corresponding percentile in the earlier decade.

the $x$-axis and the 2017 value is indicated with a vertical dashed line. This value is in the $99.1 \%$ tail of the 2010 2020 decade's probability density estimate. The 99.1 percentile of the 1960-1970 probability density estimate corresponds to a fire 9 times smaller, indicated by the solid vertical line. The shading around this line indicates the variability in the estimate, obtained using the bootstrap simulation method (Efron, 2013), and is the source of the 7-11 conclusion. The increase in the estimated magnitude of the area burned in the decade 2010-2020 is attributed to anthropogenic influence, as the earlier decade is assumed to represent scenarios in the absence of human-induced warming.

This short summary necessarily omits many details of the science and methodology, which are carefully described in the paper and supplementary material. The climate data was obtained from an ensemble of 50 simulated climate series provided by the Canadian Regional Climate Model, which in turn is derived from the Canadian Earth System Model of climate, downscaled to the region of British Columbia of interest. Surface measurements of mean air temperature and precipitation anomalies were created from a variety of sources, including the National Aeronautics and Space Administration, the MultiSourced Weighted Ensemble Precipitation, DataBC, University of East Anglia, BC Hydro, Rio Tinto, and others. When needed, spatial measures were interpolated across 
the area between weather stations using statistical methods. Area burned was estimated through regression using observational data and published fire weather indices. The authors also investigated temperature and precipitation anomalies and fraction of risk of fire attributable to human-induced warming, and discussed thoroughly the assumptions and potential limitations of their work.

This work exemplifies data science in action: climate models are built on a sophisticated mix of simulation, climate science, physics, and mathematics. The amalgamation of observational data sets from various sources uses mathematical and statistical models to create a smooth field of measurements across the area of interest. Statistical methods include regression models, density estimation, bootstrap inference, spline smoothing, and more. It is not conceivable that such a study would have been feasible even twenty years ago, yet such studies are now commonplace. At the same time, while there are many sources of data, and the datasets are complex, they are not really very big. Their structure is much more interesting and relevant than their size.

In late 2019 and early 2020 the world's attention was gripped by a series of bushfires in Australia, some of which threatened major cities, including Sydney and Canberra. Many headlines attributed the fires to climate change, noting that during the same period Australia recorded its hottest ever land temperature. In November 2019 the BBC noted "the science around climate change is complex-it's not the cause of bushfires but scientists have long warned that a hotter, drier climate would contribute to Australia's fires becoming more frequent and more intense." The article quoted Dr. Richard Thornton, chief executive of the Bushfires \& Natural Hazards Co-operative Research Centre: "We find it very difficult in general to attribute climate change impacts to a specific event, particularly while the event is running." In contrast to this, on January 2 2020, the Washington Post wrote: "For the first time, scientists have detected the 'fingerprint' of human-induced climate change on daily weather patterns at the global scale. ... If verified by subsequent work, the findings ... would upend the long-established narrative. ... The new study ... uses statistical techniques and climate model simulations." The worked referred to is Sippel et al. (2020), who claimed that climate change could indeed be identified in a single day of global weather. This is not exactly the same as attribution of a single event, but it does provide a counterpoint to the distinction made until now between climate and weather. If this result is verified in other studies, our understanding of the impacts of climate change will be further refined.

3.2. Art and life. In late 2019 the New York Times ran an article with the headline "Another Benefit of Going to
Museums? You May Live Longer." The paper also put out a tweet with a similar message: "Want to live longer? Try going to the opera." The backlash was swift, as it often is on Twitter, with comments like "Want to live longer? Try driving a BMW 7," "Rich people live longer. Who knew?," and so on. The mantra of statistical science, correlation is not causation, seems to have eluded the headline writers, although the text of the news article was more circumspect, mentioning things like "the study controlled for socioeconomic factors like ... income, education level, and mobility." The article also stated that "the researchers combed through the data they had collected to search for patterns" a sentence that should ring alarm bells with its suggestion of data-dredging. ${ }^{5}$

This article was reporting on a study published in the British Medical Journal (Fancourt \& Steptoe, 2019), and it is noteworthy that the authors used "association" in the title; often titles of press releases use language that implies causation, even when the article does not. The data used was collected as part of the English Longitudinal Study on Aging (Steptoe et al., 2013), which started in 2002, and is part of an international effort. ${ }^{6}$ Surveys are carried out each two years and the data for this study came from the 2nd wave of responses, collected in 2004-2005. It was a prospective observational study, which followed people over time, so is not plagued with recall bias. It is however observational so any association between arts engagement and longevity has two potential causal directions. The best that can be established is that the association between the two is not explained away by a more direct cause-income and health being two obvious candidates.

The response of interest was survival time, recorded up to March, 2018, and the research question was to investigate any association with "receptive arts engagement," which was defined by the authors as "including going to the theatre, concerts, opera, museums, art galleries, and exhibitions" (Fancourt \& Steptoe, 2019). Participants were also measured on a large number of demographic, socioeconomic, and health-related variables, including for example sex, age, education, employment status, eyesight, chronic illnesses, social engagement measures, and more. The unadjusted data is displayed in Table 1 , adapted from Table 2 in Fancourt \& Steptoe (2019), which indeed shows a strong association between mortality and arts engagement. This does not take into account survival time, nor other covariates.

The more relevant analysis uses survival time as a response, and arts engagement along with a number of other explanatory variables in a regression model, to enable

${ }^{5}$ In fact there is no evidence of data-dredging in the published paper, although a cursory glance at the paper might give that impression.

${ }^{6}$ The US Health and Retirement study is in the same network. 
Table 1. Receptive Arts Engagement. Summary data from Fancourt \& Steptoe (2019), Table 1. The column categories refer to engagement with the arts, as described in the methods section of their paper.

\begin{tabular}{llll}
\hline & Never & Infrequently & Frequently \\
\hline Died & $837(47.5 \%)$ & $809(26.6 \%)$ & $355(18.6 \%)$ \\
Survived & 925 & 2233 & 1551 \\
\hline Total & 1762 & 3042 & 1906
\end{tabular}

adjustment of the observed mortality rates for potential confounding variables. The proportional hazards model (Cox, 1972) is widely used for this purpose, and can properly use information from survival times that are censored because the respondents were still alive at the time of data analysis. As there were 6,710 people in the dataset, the authors were able to adjust for a large number of potential confounders.

The authors concluded that even after adjustment for various socioeconomic and health measurements, infrequent engagement with the arts was associated with a reduction in risk of mortality by $14 \%(4-23 \%)$, and that frequent engagement was associated with a reduction of $31 \%$ $(20-41 \%)$, both of these relative to the "never engaged" group. The intervals are 95\% confidence intervals, and because these confidence intervals do not include the null value 1 , or $100 \%$, the differences would conventionally be described as "statistically significant," as the $p$-value is less than 0.05 . Reporting only that $p<0.05$ has repeatedly come under considerable criticism; see $\$ 4$ below. Here however the authors reported both survivor functions and confidence intervals, which is much more informative.

The validity of the proportional hazards assumption in their modelling was checked by analysis of residuals, which compare the model predictions to the observed data. The analysis was weighted using techniques of survey sampling, in order to relate the people in the survey to the population of interest. A number of sensitivity analyses were performed, and a measure of the proportion of the survival discrepancy due to the confounders was calculated: it was $41 \%$, indicating that nearly $60 \%$ of the positive effect of arts engagement was not explained by confounding factors. The study also found a dose-response effect, as arts engagement was categorized as never, infrequently, and frequently. The authors were careful to note in the discussion that their study "suggests that receptive arts engagement could have independent longitudinal protective associations with longevity" (my emphasis) and that "A causal relationship cannot be assumed, and unmeasured confounding factors might be responsible for the association" (Fancourt \& Steptoe, 2019).

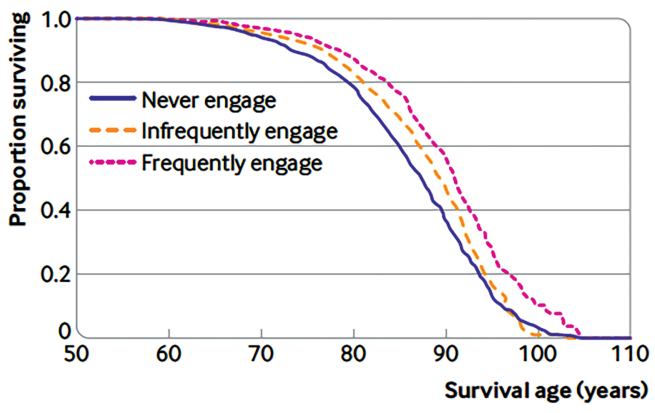

Fig 2 | Survivor function, which shows survival age by frequency of receptive arts engagement when adjusting for demographic, socioeconomic, health related, behavioural, and social confounding factors

Figure 4. Figure 2 of Fancourt \& Steptoe (2019), showing the estimated survival probabilities stratified by arts engagement. These were estimated after adjusting for confounding variables related to health, socioeconomic status, social engagement, and so on. The relative risk (instantaneous failure rate) is estimated to be $31 \%$ lower in the "frequently engage" group, and $14 \%$ lower in the "infrequently engage" group, relative to the "never engage" group.

Although I began my reading of the study aligned with the dismissive comments on Twitter, my conclusion is that this was an exceptionally careful analysis, built on a carefully collected set of data. The authors were circumspect in their claims, and were very thorough in assessing their assumptions and the sensitivity of their conclusions. Dr. Fancourt herself tweeted "Confounders obviously a big challenge but results consistent in well adjusted models \& sensitivity analysis." While undoubtedly not the last word on the subject, this is a convincing and careful study.

It is also a study firmly in the realm of statistical science and small data, and is included to emphasize that there is much to learn from small data sets of high quality, using an arsenal of well-established statistical methods.

\section{Reproducibility and Visualization}

These two important pieces of the data science workflow in Figure 2 are starting to be addressed more systematically in statistics and data science programs and courses. Reproducibility is emphasized as a model of good practice: with the same data and following the steps outlined by the original investigators, another investigator should be able to obtain the same results. This is harder than it sounds, but there are several software systems available now, such as RStudio projects and Jupyter notebooks that are designed to make it easier to ensure reproducibility of statistical analyses; resources such as Github make it easier to share code and data, and suites of tools have been developed especially for "data wrangling" (for example, Wickham \& Grolemund, 2016). Replicability is a more demanding standard, that an independent investigation of the same question, with possibly different data, leads to 
results consistent with the first investigation. This is the cornerstone of the scientific method, but in the data science context it would normally include a requirement that the same or very similar statistical analyses were carried out in both cases. ${ }^{7}$

The examples above were selected to illustrate highquality statistical analysis on carefully described data, each making an important contribution to science or social science, respectively, and each setting their work in context and addressing its limitations. In principle the results are reproducible, as the methods and sources of data are clearly delineated, but the effort in doing so would be substantial. Replication of the results on similar data might entail, for example, an attribution study for the 2018 wildfire season in British Columbia for Example 1, or a comparable analysis of data from one of the consortium partners for Example 2.

There are unfortunately many further examples of more dubious quality, and there are even books written on the topic (Bergstrom \& West, 2020). A haphazard sample of headlines that I collected in preparation for the presentation included "Quitting Facebook could change your life," "Claims about a treatment for Alzheimer's should be treated with caution," "Can intermittent fasting reset your immune system?"

Much discussion has centred around the contribution of $p$-values to a decline in quality. The $p$-value itself is not to blame-it is a well-defined probability that describes how unusual (or not) the observed data is, under assumptions about the underlying processes that generated the data. These assumptions usually consist of a statistical model (which may or may not be appropriate), and within that model, a working assumption that the effect one hopes to find is, in fact, absent-what statisticians call a "null hypothesis." In the art appreciation example above, the null hypothesis is that receptive engagement with the arts has no association with survival. A small $p$-value indicates that the data are not consistent with the null hypothesis, so has a weak parallel to a proof by contradiction; either the null hypothesis is incorrect, or we have observed an event (set of data) of very low probability. In practice the dataset is typically unusual in only one respect-for example often the mean value of a response is larger than would be expected under the null hypothesis.

There are many sources of advice for increasing the strength of a statistical conclusion by supplementing $p$ values with estimates of effect size (for example, the 31\% reduction in risk illustrated in Figure 4 ), for providing confidence intervals for effect sizes (for example, the 7-11fold increase in area burned illustrated in Figure 3), for

\footnotetext{
7These meanings of "reproducibility" and "replicability" follow the 2017 ASA guidelines, but usage is not standardized across all fields.
}

undertaking sensitivity analyses, and more; see for example Kass et al. (2016). It has been customary, since Fisher suggested it more than 100 years ago, to use $p=0.05$ as an arbitrary dividing line between "statistically significant" $(p<0.05)$ and "not statistically significant" $(p>0.05)$, but any scientific conclusions based on such a simple dichotomy should be regarded with considerable skepticism. The validity of scientific results is hampered by the rote use of any tool, and $p$-values are just one of these. Unfortunately $p$-values are both widely used and widely misunderstood. Alarm bells should ring when a study of particular interest to you or to society reports a result that was "small, but statistically significant." Most science is more complex than that.

Visualization of statistical analyses and data summaries has been an under-emphasized sideline in statistical training for many years. Tufte's classic book (Tufte, 1983) elegantly set out a number of principles for graphical display, and early work by Cleveland (1985), Wilkinson (1999), and many others put the construction of statistical graphics on firm intellectual ground. Data visualization is now a thriving discipline that blends computer science, statistical science, and art, and the advances in the past twenty years in journalism and on web pages have been stunning. Several media outlets now have large visualization departments. Among many aggregator sites that emphasize data visualization, flowingdata. com is a particularly interesting resource. There are also plenty of unhelpful and downright misleading visualizations; for example Lumley's blog shows how some circulating maps of the bushfires in Australia in 2020 were misleading.

\section{Conclusion}

Statistical science and data science are in a phase of very high public interest and accompanying hype. This tends to get bound up with the similarly high interest in machine learning and artificial intelligence, which are undoubtedly seeing even more hype. It seems that every day there are remarkable advances in some aspect of datadriven discovery-improvements in image processing, discoveries in astronomy, identification of genetic signatures, and so on. One popular pronouncement was "data is the new oil," intended one presumes to send the message that companies that learn how to jump on the data bandwagon will prosper, and the sooner one jumps, the better.

This paper is entitled "In Praise of Small Data" at least partly to attempt to dispel the data-deluge description that is the basis for a great deal of this hype. Scientists have always had more data than they quite knew what to do with even when that data was being recorded with pencil and paper-it is in the nature of scientific discovery to work at the boundary of what is possible. 
If data science is indeed a new discipline and area of research, and not simply a new program of study and a new job description, the size of the data will be one of the least interesting aspects. Even in fields such as high-energy particle physics, where terabytes of data are collected nearly continuously, the most important scientific questions rely on the study of a few very rare events. The investigation of the relationship between climate change and wildfire described in $\$ 3.1$ (Kirchmeier-Young et al., 2019) relied on data from a large number of sources-governments around the world, data from mining and hydro-electric companies, and more. But the conclusions were based on just 500 observations -50 climate simulations in two 10 -year periods. The study of art and life (Fancourt \& Steptoe, 2019) described in $\$ 3.2$ relied on responses from 6,710 participants of an ongoing survey, and was able to provide reasonably confident conclusions about the population of Britain, thanks to the quality of the survey design and data collection. Quality is much more important than quantity, which should be obvious, but bears repeating, especially as there are many commercial enterprises emphasizing quantity instead.

It is an exciting time to be working with data-there is more enthusiasm for, and interest in, statistical science than I have seen in my career. The emerging world of data science will I hope combine the best of statistics, mathematics, information science, and social science to raise the bar for scientific advances, social improvements, and policy decisions. Clear understanding of the strengths and limitations of data-intensive research will be our best resource.

ACKNOWLEDGMENTS. I am grateful to the American Mathematical Society for giving me the opportunity to think about these issues, and to summarize my thoughts in this paper. I would like to thank the reviewers of an earlier version for prompt and helpful comments. This work was partially funded by the Natural Sciences and Engineering Research Council of Canada.

\section{References}

[1] C. T. Bergstrom and J. D. West, Calling Bullshit: The Art of Skepticism in a Data-Driven World, Random House, New York, 2020.

[2] Steve Brooks, Andrew Gelman, Galin L. Jones, and XiaoLi Meng (eds.), Handbook of Markov chain Monte Carlo, Chapman \& Hall/CRC Handbooks of Modern Statistical Methods, CRC Press, Boca Raton, FL, 2011. MR2742422

[3] George Casella and Edward I. George, Explaining the Gibbs sampler, Amer. Statist. 46 (1992), no. 3, 167-174, DOI 10.2307/2685208 MR1183069
[4] W. S. Cleveland, The Elements of Graphing Data, Hobart Press, New Jersey, 1985, updated 1994.

[5] D. R. Cox, Regression models and life-tables, J. Roy. Statist. Soc. Ser. B 34 (1972), 187-220. MR341758

[6] D. R. Cox and Christl A. Donnelly, Principles of Applied Statistics, Cambridge University Press, Cambridge, 2011. MR2817147

[7] David Donoho, 50 years of data science, J. Comput. Graph. Statist. 26 (2017), no. 4, 745-766, DOI 10.1080/10618600.2017.1384734. MR3765335

[8] C. Dwork, A. Smith, T. Steinke, and J. Ullman, Exposed! A survey of private attacks on public data, Annual Review of Statistics and its Application 4 (2017), 61-84.

[9] Bradley Efron, A 250-year argument: belief, behavior, and the bootstrap, Bull. Amer. Math. Soc. (N.S.) 50 (2013), no. 1, 129-146, DOI 10.1090/S0273-0979-2012-01374-5 MR2994997

[10] D. Fancourt and A. Steptoe, The art of life and death: 14 year follow-up analyses of associations between arts engagement and mortality in the English Longitudinal Study of Aging, British Medical Journal 367 (2019). https://www.bmj . com/content/367/bmj . 16377

[11] S. Geman and D. Geman, Stochastic relaxation, Gibbs distributions, and the Bayesian restoration of images, IEEE Transactions on Pattern Analysis and Machine Intelligence, PAMI-6 (1984), 721-741, DOI 10.1109/TPAMI.1984.4767596

[12] M. B. Hawes, Implementing differential privacy: seven lessons from the 2020 US Census, Harvard Data Science Review 2 (2020). https://doi . org/10.1162/99608+92 $.353 c 6 f 99$

[13] R. E. Kass, B. S. Caffo, M. Davidian, X.-L. Meng, Bin Yu, and N. Reid, Ten simple rules for effective statistical practice, PLOS Computational Biology (2016), https: //doi .org /10.1371/journa7.pcbi.1004961

[14] M. C. Kirchmeier-Young, M. P. Gillet, F. W. Zwiers, A. J. Cannon, and F. S. Anslow, Attribution of the influence of human-induced climate change on an extreme fire season, Earth's Future 7 (2019). https://doi.org/10 $.1029 / 2018 \mathrm{EF} 001050$

[15] X.-L. Meng, Five immersive 3D surroundings of data science, Harvard Data Science Review 1 (2019). https:// doi .org/10.1162/99608f92 . ab81d0a9

[16] Cathy O'Neil, Weapons of Math Destruction: How big data increases inequality and threatens democracy, Crown, New York, 2016. MR3561130

[17] Jerome P. Reiter, Differential privacy and federal data releases, Annu. Rev. Stat. Appl. 6 (2019), 85-101, DOI 10.1146/annurev-statistics-030718-105142 MR3939513

[18] C. Rudin, C. Wang, and B. Coker, The age of secrecy and unfairness in recidivism prediction, Harvard Data Science Review 2 (2020). https: //doi . org/10.1162/99608f92 $.6 \mathrm{ed} 64 \mathrm{~b} 30$

[19] S. Sippel, N. Meinshausen, E. M. Fischer, E. Székely, and R. Knutti, Climate change now detectable from any single day of weather at global scale, Nature Climate Change 
10 (2020), 35-41. https://doi .org/10.1038/s41558 $-019-0666-7$

[20] A. Steptoe, E. Breeze, J. Banks, and J. Nazroo, Cohort profile: the English longitudinal study of ageing, International Journal of Epidemiology 42 (2013), 1640-1648. https://doi.org/10.1093/ije/dys168

[21] E. Tufte, The Visual Display of Quantitative Information, Graphics Press, Cheshire, CT, 1983, 2nd edition 2001.

[22] H. Wickham and G. Grolemund, $R$ for Data Science: import, tidy, transform, visualize and model data, O'Reilly, Sebastopol, CA, 2016.

[23] Leland Wilkinson, The Grammar of Graphics, 2nd ed., Statistics and Computing, Springer, New York, 2005. With contributions by Graham Wills, Dan Rope, Andrew Norton, and Roger Dubbs. MR2161269

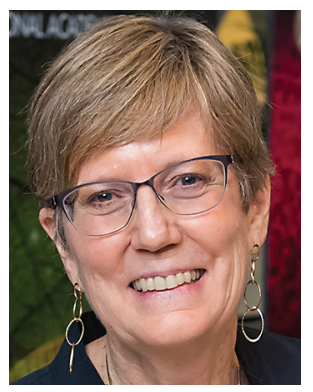

Nancy Reid

\section{Credits}

Figures 1 and 2 are courtesy of Nancy Reid.

Figure 3 is courtesy of AGU.

Figure 4 is courtesy of BMJ Publishing Group Ltd.

Photo of Nancy Reid is courtesy of Kevin Allen.

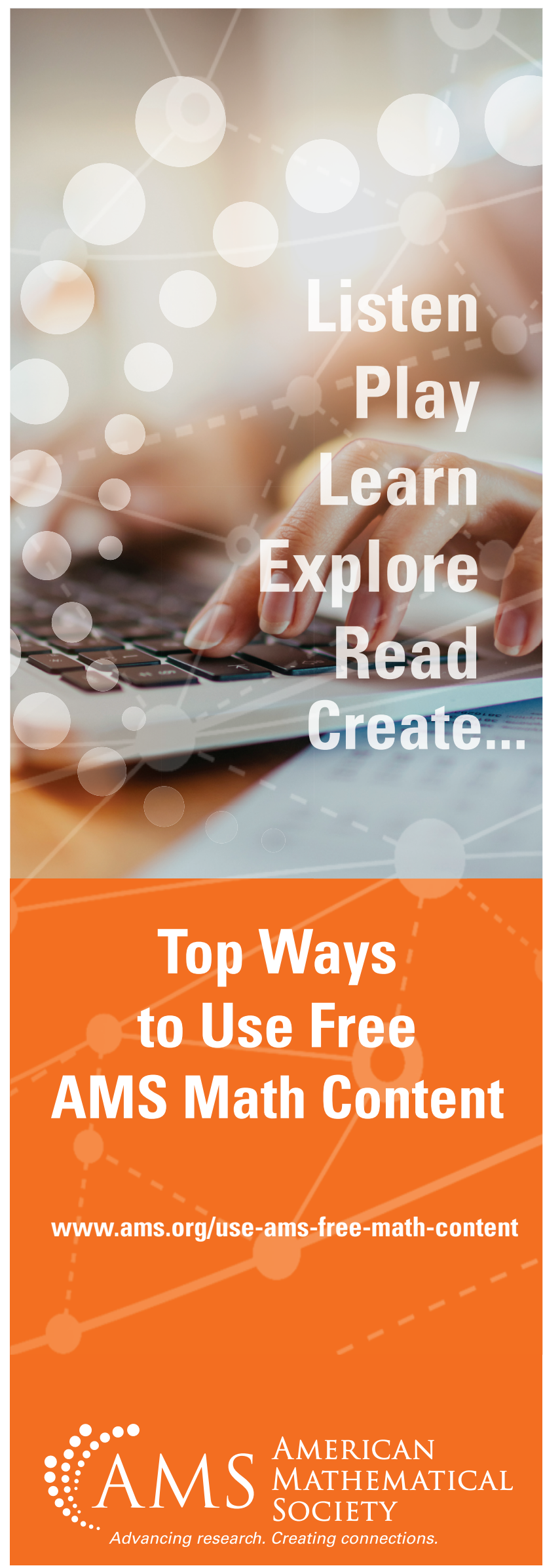

